\title{
Bipolar patient's functioning and Well-being in Argentina
}

\section{Objectives}

To determine the psychosocial functioning (FAST, Scale), and subjective well-being level (WHO-5 Scale) in Argentinian stable patients with bipolar disorder.

\section{Background}

Bipolar Disorder (BD) is a serious mood illness, characterized by mania or hypomania episodes, alternating or combined with depressive episodes.

The risk study of anxiety and mood disorders conducted in the United States showed a lifetime prevalence of $4.1 \%$ for BD. In Argentina, an annual prevalence of $2 \%$ is estimated for BD I and II.

BD seems to be more than a mood disorder. Several studies have shown that cognitive decline is a key feature of bipolar disease. In bipolar patients both functionality and quality of life are affected. In furtherance, deterioration of the level of subjective well-being can be observed.

\section{Materials and Methods}

The signature of the informed consent was required. Patients and controls completed the questionnaires to determine the Psychosocial Functionality (FAST) and the subjective well-being index (WHO-5).

Observational cross-sectional study of patients with a diagnosis of bipolar disorder undergoing treatment at CEMIC University Institute (Service of Psychiatry) and FUBIPA - Argentine Bipolar FoundationSubjects were recruited between January 2017 and September 2018. The study included subjects older than 18 years with established diagnosis of bipolar spectrum disorder according to the DSM-5 criteria, who were stable at recruitment time [Quick Inventory of Depressive Symptoms (QID-S-16) $\leq 10$ points; and Young's Scale $\leq$ 11 points], and controls. The study subjects were asked to sign an informed consent form. Patients and controls had to fill in questionnaires to determine their Psychosocial Functionality (FAST) and subjective well-being index (WHO-5).

The FAST was developed by the Bipolar Disorder Program, Barcelona, Spain, to assess functional impairment focused on the main problems experienced by the mentally ill, including bipolar patients. The WHO- 5 is a self-administered scale only containing five positively phrased items.
Variables such as age, gender, educational level, occupation, diagnosis, type of episode, age at onset, years of illness, hospitalizations, and suicide attempts were collected.

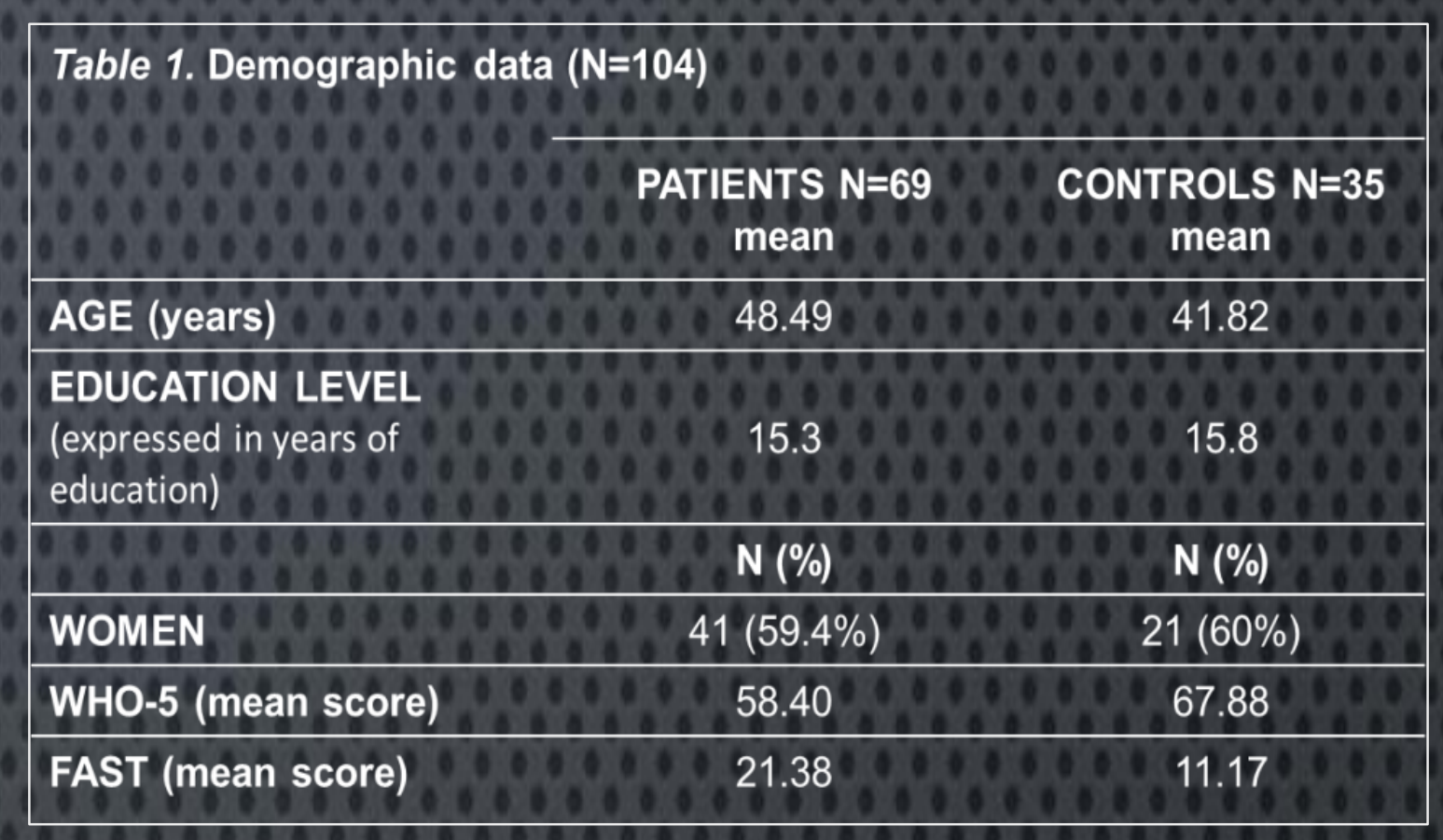

\section{Results}

A sample of 105 subjects was obtained: 70 patients with some diagnosis of bipolar disorder spectrum and 35 healthy controls. One patient with a QID-S $>10$ was excluded. Average age was 49 and 42 years, respectively; the educational level was 15 years for both groups, and $60 \%$ of the total sample were women (equal in both groups). Table 1. The FAST scale mean score was 21.38 for patients and 11.17 for controls, which represented a statistically significant difference $(T=-4.25, p<0.001)$. The WHO- 5 scale means were 58.40 and 67.88 for bipolar patients and controls, respectively. This difference was statistically significant $(t=2.70, p=$ 0.0081). Graphic 1.

\section{Conclusions}

Bipolar patients, though stable, had a worse psychosocial functionality and subjective well-being than non-bipolar controls.

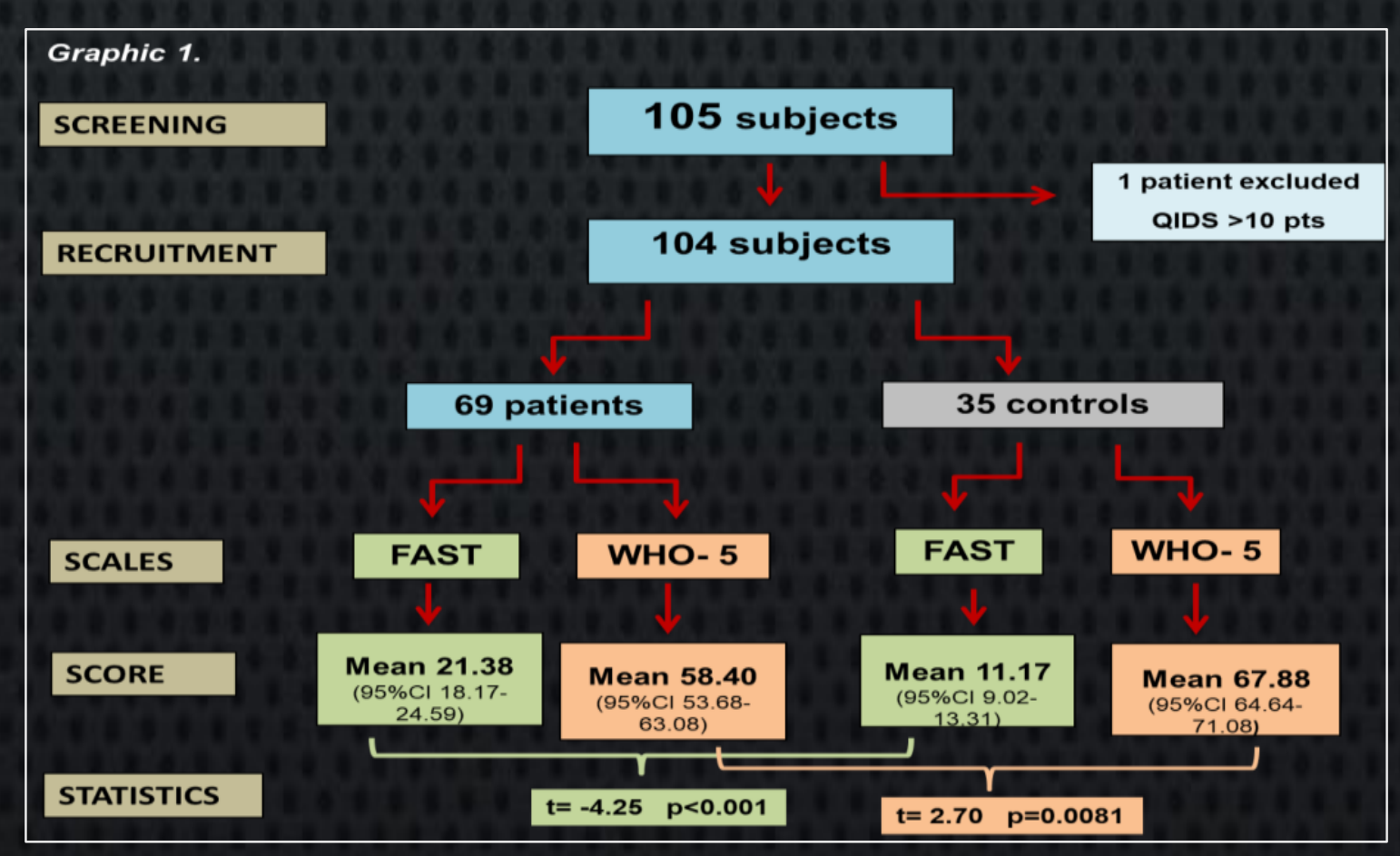

\section{Bibliograpy}

